EPJ Web of Conferences 16, 01004 (2011)

DOI: $10.1051 /$ epjconf/20111601004

(C) Owned by the authors, published by EDP Sciences, 2011

\title{
The observation of exoplanet transit events in China
}

\author{
X.-B. Wang ${ }^{1, a}$, S.-H. Gu ${ }^{1}$, A. Collier Cameron ${ }^{2}$ \\ and X.-S. Fang ${ }^{1}$ \\ ${ }^{1}$ National Astronomical Observatories/Yunnan Observatory, Chinese Academy of Sciences, \\ Kunming 650011, China \\ ${ }^{2}$ School of Physics and Astronomy, University of St. Andrews, Fife KY16 9SS, UK
}

\begin{abstract}
We have carried out a research project on the exoplanet transit events at Yunnan Observatory. By using CCD cameras attached to $1 \mathrm{~m}$ telescope of Yunnan Observatory and $85 \mathrm{~cm}$ telescope of Xinglong station, NAOC, a group of exoplanet systems with transit events have been observed photometrically. By means of MCMC method, the preliminary results of the systems WASP-11 and XO-2 are derived. Finally, we give out the future plan on this research topic in China.
\end{abstract}

\section{INTRODUCTION}

The photometric observation for an exoplanet transit event [1] can provide us the radius of exoplanet and inclination of the orbital plane. Combining with mass function of the exoplanet from the radial velocity measurements, we can determine its density. All above information is very important for understanding the formation and evolution of the exoplanet system.

Up to now, there are many survey projects for detecting the exoplanet transit events, like SuperWASP [2], XO [3], HATNet [4], etc. Based on these survey observations, 62 transiting planetary systems have been discovered. By monitoring some of these transiting exoplanet systems, we have a chance to find out some clues on the dynamic evolution of the planetary systems. On the other hand, the monitoring for the changes on orbital inclination, transit time and duration makes it possible to detect other low-mass exoplanet in the system.

Here, we present some preliminary results on the follow-up observations of exoplanet transit events at Yunnan Observatory. Then, we shall discuss the future plan for this topic in China.

\section{OBSERVATION}

Our follow-up observations for transit events of exoplanets were begun in 2006. After that, we observed several transit events each year. The instruments used in the observations are $1 \mathrm{~m}$ telescope with $1 \mathrm{Kx} 1 \mathrm{~K}$, $2 \mathrm{Kx} 2 \mathrm{~K}$ CCD cameras of Yunnan Observatory and $85 \mathrm{~cm}$ telescope with $1 \mathrm{Kx} 1 \mathrm{~K}$ CCD camera [5] of Xinglong station, NAOC. In all observations, the R filter was employed.

During our observations, the targets were selected from the following survey projects:

SuperWASP project: WASP-1b, WASP-2b,WASP-11b, etc.

$\mathrm{XO}$ project: $\mathrm{XO}-2 \mathrm{~b}, \mathrm{XO}-3 \mathrm{~b}$, etc.

HATNet project: HAT-P-1b, HAT-P-9b, etc.

CoRoT project: CoRoT-Exo-1b, etc.

ae-mail: wangxb@ynao.ac.cn

This is an Open Access article distributed under the terms of the Creative Commons Attribution-Noncommercial License 3.0, which permits unrestricted use, distribution, and reproduction in any noncommercial medium, provided the original work is properly cited. 
EPJ Web of Conferences

Table 1. The optimal parameters derived for WASP-11 system using the MCMC method.

\begin{tabular}{|c|c|}
\hline Parameter & Value \\
\hline Transit epoch $T_{c}$ [HJD] & $2454793.1887^{+0.0002}-0.0002$ \\
\hline Transit depth $\Delta F$ & $0.0168^{+0.0002}-0.0001 \mathrm{mag}$ \\
\hline Transit width $t_{T}$ & $0.0968+0.0003-0.0004$ days \\
\hline Impact parameter $b$ & $0.0077^{+0.0719}{ }_{--0.0072} \mathrm{R}_{*}$ \\
\hline Orbital separation $a$ & $0.04308539+8.8 E-07-2.49 E-06 \mathrm{AU}$ \\
\hline Orbital inclination $i$ & $89.964^{+-0.033}-0.338$ degrees \\
\hline Stellar radius $R_{*}$ & $0.757^{+0.004}-0.002 \mathrm{R}_{\odot}$ \\
\hline Planet radius $R_{p}$ & $0.956^{+0.009}{ }_{-0.004} \mathrm{R}_{J}$ \\
\hline Stellar mass $M_{*}$ & $0.769999997+5.3 E-08{ }_{-4.2 E-08} \mathrm{M}_{\odot}$ \\
\hline Planet mass $M_{p}$ & $0.525624^{+1.1 E-05}-1.1 E-05 \mathrm{M}_{J}$ \\
\hline
\end{tabular}

Table 2. The optimal parameters derived for XO-2 system using the MCMC method.

\begin{tabular}{|c|c|}
\hline Parameter & Value \\
\hline Transit epoch $T_{c}$ [HJD] & $2454804.3292^{+0.0003}-0.0002$ \\
\hline Transit depth $\Delta F$ & $0.0098^{+0.0003}-1.7 E-05 \mathrm{mag}$ \\
\hline Transit width $t_{T}$ & $0.0998^{+0.0006}-0.0005$ days \\
\hline Impact parameter $b$ & $0.0459^{+0.2644}{ }_{--0.0206} \mathbf{R}_{*}$ \\
\hline Orbital separation $a$ & $0.03743901+1.27 E-06-9.200 E-05 \mathrm{AU}$ \\
\hline Orbital inclination $i$ & $89.689^{+-0.140}-1.895$ degrees \\
\hline Stellar radius $R_{*}$ & $0.950^{+0.047}--0.001 \mathrm{R}_{\odot}$ \\
\hline Planet radius $R_{p}$ & $0.917^{+0.059}{ }_{--0.002} \mathrm{R}_{J}$ \\
\hline Stellar mass $M_{*}$ & $0.989999992+7.4 E-08{ }_{-5.0 E-08} \mathrm{M}_{\odot}$ \\
\hline Planet mass $M_{p}$ & $0.6775^{+0.0002}-0.0006 \mathrm{M}_{J}$ \\
\hline
\end{tabular}

\section{DATA REDUCTION}

The observed CCD images are reduced by using IRAF package, including the bias subtraction, flatfielding and cosmic ray removal. The instrumental magnitudes of the planetary systems and comparison stars in the field of view are measured by using APPHOT sub-package of IRAF for the targets located in the uncrowded field. If the targets are in the crowded field, we employ the image subtraction method [6] to derive the light curves of transit events.

For the light curves derived by using aperture photometry, we remove the systematic errors by using coarse decorrelation method [7] and SysRem algorithm [8].

\section{LIGHT CURVE ANALYSIS}

Here, we give two examples of transiting light curve analysis for the systems WASP-11 and XO-2. We model the flux of the transiting systems with the parameters $\left\{T_{c}, p, \Delta F, t_{T}, b, M_{*}\right\}$ considering the 4-coefficient limb-darkening law of Claret [9]. The limb darkening coefficients for the R-band are interpolated from Claret's table according to the effective temperature of the host stars. The basic parameters of the host stars are adopted from the recent relative results $[10,11]$. All observed data points are involved in MCMC (Markov Chain Monte Carlo) analysis to search the optimal parameters $\left\{T_{c}, p, \Delta F, t_{T}, b, M_{*}\right\}$ under the condition of minimizing the quantity $\chi^{2}$ [12]. Here, the chi-squared $\chi^{2}=\sum_{j}\left(m_{j}-\mu_{j}\right)^{2} / \sigma_{j}^{2}, m_{j}$ and $\mu_{j}$ are observed and model flux, respectively. The final results are listed in Table 1 and 2, the relative fitting and other information are displayed in Fig. 1 and 2. 
Research, Science and Technology of Brown Dwarfs and Exoplanets
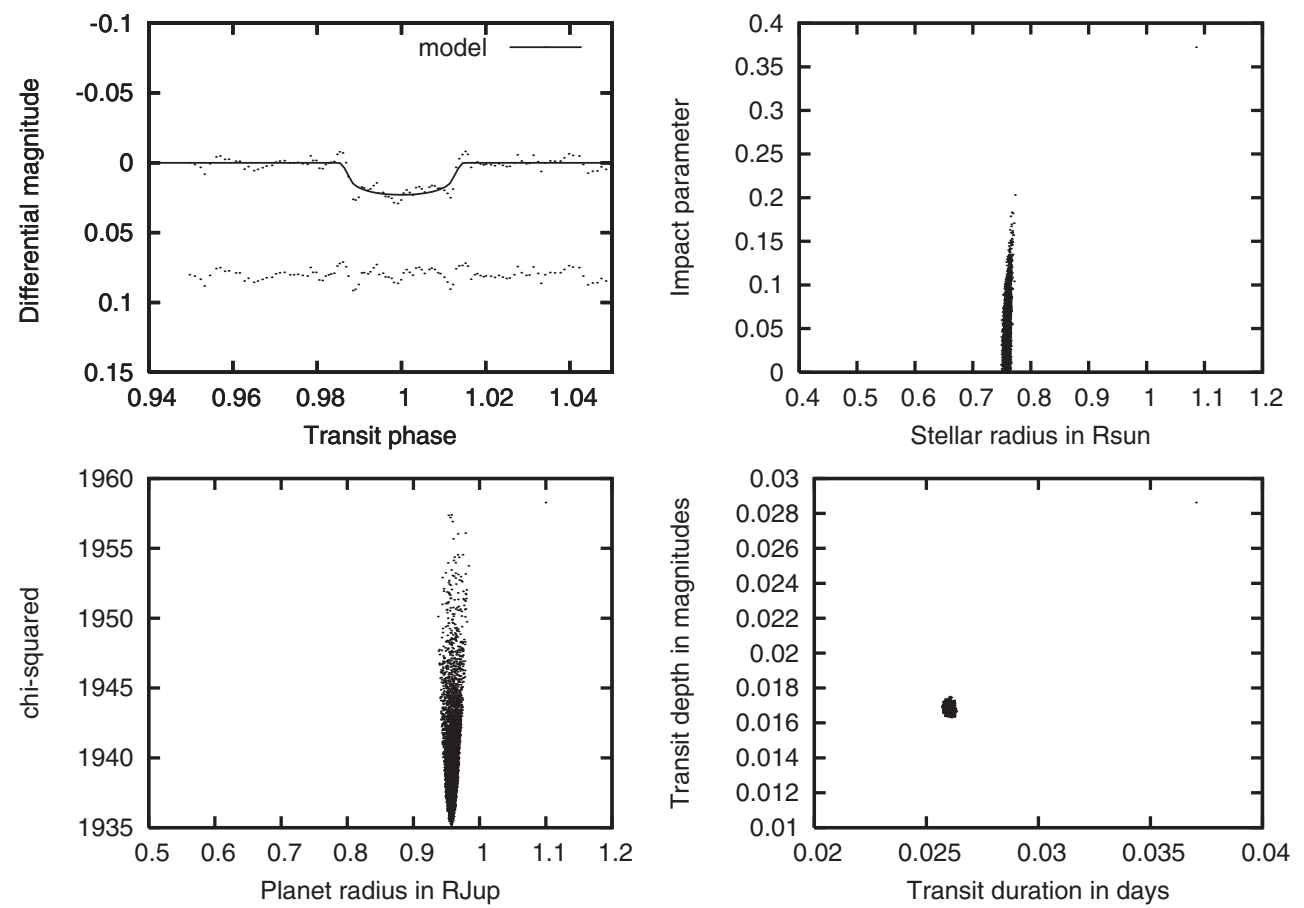

Figure 1. Upper-left panel: Light curve of WASP-11 system and fitting. Lower-left panel: The radius of planet (in unit of radius of Jupiter) vs chi-squared. Upper-right panel: The relation between radius of star and impact parameter. Lower-right panel: The relation between transit duration and transit depth.
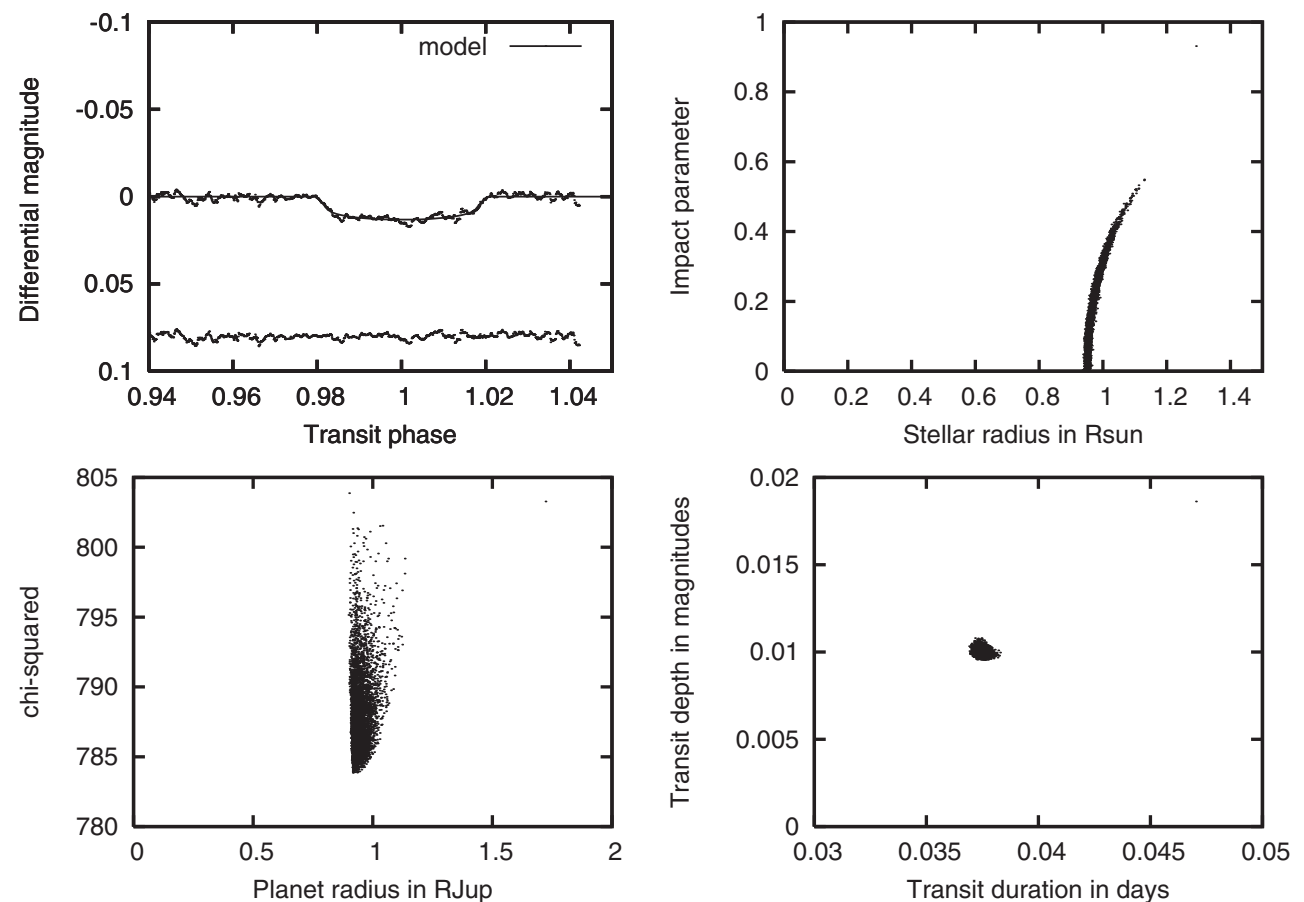

Figure 2. The same as Fig. 1, but for XO-2 system. 


\section{FUTURE PLAN}

Now, we have a new $1.3 \mathrm{Kx} 1.3 \mathrm{~K}$ CCD camera attached to $2.4 \mathrm{~m}$ telescope at Lijiang station of Yunnan Observatory. In 2010, we shall build other three instruments for $2.4 \mathrm{~m}$ telescope, which are YFOSC with a $4 \mathrm{Kx} 4 \mathrm{~K}$ CCD camera and FOV of $9^{\prime} \times 9^{\prime}$, Mosaic CCD camera with a dimension of $6 \mathrm{Kx} 6 \mathrm{~K}$ and FOV of 9'x9', LiJET(LiJiang Exoplanet Tracker) for accurate radial velocity measurement.

By using above new instruments, we prepare to carry out the following research projects for the study of exoplanet:

1. In order to improve the accuracy of transit light curves, we shall perform some defocus observations for the transit events of some exoplanets using three new CCD cameras.

2. We shall start an open cluster transit survey by using YFOSC CCD camera and Mosaic CCD camera so as to discover new transiting exoplanet candidates. Then, we shall use LiJET to do follow-up confirmation for the brighter ones of them.

This work is supported by NSFC under grant No.10673027.

\section{References}

[1] Charbonneau, D. et al., ApJ 529, (2000) 45

[2] Pollacco, D.L. et al., PASP 118, (2006) 1407

[3] McCullough, P.R. et al., PASP 117, (2005) 783

[4] Bakos, G. et al., PASP 116, (2004) 266

[5] Zhou, A.-Y. et al., RAA 9, (2009) 349

[6] Alard, C., Lupton, R.H., ApJ 503, (1998) 325

[7] Collier Cameron, A. et al., MNRAS 373, (2006) 799

[8] Tamuz, O., Mazeh, T., Zucker, S., MNRAS 356 (2005) 1466

[9] Claret, A., A\&A 363, (2000) 1081

[10] West, R.G. et al., A\&A 502, (2009) 395

[11] Burke, C.J., ApJ 671, (2007) 2115

[12] Collier Cameron, A. et al., MNRAS 380, (2007) 1230 